\title{
FINE-SCALE ANISOTROPY OF THE COSMIC MICROWAVE BACKGROUND RADIATION
}

\author{
PAUL E. BOYNTON \\ Dept. of Astronomy, University of Washington, Seattle, Wash., U.S.A.
}

\section{Introduction}

In keeping with the title of Symposium No. 63, I would like to confront the assembly with a short review of observational upper limits on the amplitude of intensity fluctuations in the microwave background radiation on angular scales of less than one degree. These fluctuations are presently of interest for at least two important cosmological concerns:

\subsection{Perturbations on the relic radiation}

Interpreted as small angular scale temperature variations on an otherwise smooth background of $2.7 \mathrm{~K}$ thermal radiation, such observations may yield information on the initial mass spectrum of density inhomogeneities which, through gravitational instability, have evolved into the highly structured universe which we presently observe. On the other hand, if primeval turbulence is responsible for the initial development of these mass associations, the velocity dispersion of the material in which the radiation was last scattered will produce doppler shift perturbations on the radiation temperature which also appear as small angular scale 'roughness' on the general background.

\subsection{DisCRETE SOURCE EFFECTS}

Clearly, even if one views the background radiation as the smooth, thermal equilibrium remnant of the Big Bang, at some level ordinary radio sources will contribute to an observed sky roughness. Thus simple discrete source confusion imposes an observational lower limit on the rms amplitude of background fluctuations. Although this is an important technical consideration, the real issue I wish to stress is cosmological: the postulate that as yet unidentified discrete sources are responsible for the total observed flux in the $2.7 \mathrm{~K}$ background. Such models have been proposed in defense of steady state cosmologies or initially cold, evolving models. Detailed assumptions are required to provide an energy spectrum consistent with observations, but to test the discrete source postulate we need only consider the fine-scale roughness in the angular distribution of radiation intensity that must follow from this model. In the low source density, high source intensity case such fluctuations would be trivially observed. In the opposite limit, because of the clustering of sources on the scale of galaxies, it is difficult to see how the effective density of remote sources can exceed the density of galaxies.

With these several possible interpretations of fine-scale fluctuations in mind, I will

M. S. Longair (ed.), Confrontation of Cosmological Theories with Obsertational Data. 163-166. All Rights Reserted. Copyright $\mathrm{O} 1974$ by the IAU. 
review very briefly the current observational results, then discuss some of these interpretations quantitatively.

\section{Observations}

Unlike the measurement of large-scale isotropy discussed by Dr Partridge in the previous paper, high angular resolution is important to this work and large aperture radio telescopes are employed at the shortest possible wavelengths to give a minimum effective beam solid angle, $\Omega$. Both beam switching and drift scan techniques have been employed. By beam switching through some angular displacement, $\phi$, and measuring the intensity (antenna temperature) differences for many such independent sample pairs, one may determine (in the absence of an observable effect) upper limits on the rms sky roughness. One is most sensitive to variations on angular scales between the characteristic values $\phi$ and $\sqrt{\Omega}$, and fluctuation limits to be considered as thermal background perturbations are generally quoted for angular scales in this range.

However, limits on unresolved fluctuations (angular scale $<\sqrt{\Omega}$ ) are of particular interest to the discrete source question. If we suppose there are on the average $N$ sources within the beam solid angle, $\Omega$, and for simplicity we assume them all to be of the same apparent brightness, the fractional fluctuation in intensity characterizing an ensemble of independent beam positions in the sky will be:

$$
\frac{(\Delta I)_{\mathrm{rms}}}{I}=\frac{1}{\sqrt{N}}=\frac{1}{\sqrt{\Omega\left(\frac{\mathrm{d} N}{\mathrm{~d} \Omega}\right)}} .
$$

Thus the projected surface density of sources is given by

$$
\frac{\mathrm{d} N}{\mathrm{~d} \Omega}=\frac{1}{\Omega}\left(\frac{I}{(\Delta I)_{\mathrm{rms}}}\right)^{2},
$$

where $(\Delta I)_{\mathrm{rms}}$ refers to noise in excess of expected receiver noise, and is best determined by the appropriate hypothesis test at a meaningful level of significance. Drift scan analysis may be discussed in similar terms.

The present status of the data is summarized in the following table.

\begin{tabular}{llllll}
\hline Observers & $\begin{array}{l}\lambda \\
(\mathrm{cm})\end{array}$ & $\begin{array}{l}\phi \\
(\operatorname{arcmin})\end{array}$ & $\begin{array}{l}\Omega \\
\left(\operatorname{arcmin}^{2}\right)\end{array}$ & $\Delta I / I$ & $\begin{array}{l}\mathrm{d} N / \mathrm{d} \Omega \\
\left(10^{11} \mathrm{sr}^{-1}\right)\end{array}$ \\
\hline Conklin and Bracewell (1967) & 2.8 & - & 300 & $<1.8 \times 10^{-3}$ & $>1$ \\
Penzias et al. (1969) & 0.35 & - & 4 & $<1.1 \times 10^{-2}$ & $>2$ \\
Parijskij and Pyatunina (1970) & 4.0 & 6 & 30 & $<2.6 \times 10^{-4}$ & $>500$ \\
Boynton and Partridge (1973) & 0.35 & 3 & 4 & $<3.7 \times 10^{-3}$ & $>20$ \\
Carpenter et al. (1973) & 3.56 & - & 18 & $<7 \times 10^{-4}$ & $>100$ \\
Parijskij (1973) & 2.8 & 3.60 & 25 & $<3 \times 10^{-5}$ & $>5000$ \\
\hline
\end{tabular}


Note that all observers have established only upper limits for the fluctuation amplitude (and consequently lower limits for the surface density of sources). No statistically significant sky roughness has yet been claimed, but from the table one sees that $\Delta I / I$ has been reduced to a value which would indicate that the Universe may be even more isotropic on small angular scales than the already impressive degree of isotropy established by the large-scale measurements reviewed in the previous paper. In this connection, notice the remarkably stringent limit posed by Dr Parijskij's most recent observation. It is regrettable that he is not able to attend the symposium to discuss this important result. The published account of this work is extremely brief and we hope that the author will soon clarify several technical points that have recently been raised.

\section{Discussion}

Interpreted as upper limits on the relict radiation perturbations by mass inhomogeneities or vortical motion in the early Universe, these values of $\Delta I / I$ are approaching the regime of significant limitations on theories of the formation of high contrast mass associations. This application of the data will be discussed later in this symposium by Drs Zel'dovich, Novikov, Ozernoy, and Silk.

In terms of limitations on discrete source models for the isotropic microwave background, one must first interpret the surface density of sources, $\mathrm{d} N / \mathrm{d} \Omega$, as a volume density through the choice of a particular cosmological model. We may consider two extremes:

(1) For Steady-State models, the required volume density of sources (implied by any of the surface density values from the table) is orders of magnitude greater than the volume density of galaxies (Hazard and Salpeter, 1969).

(2) Smith and Partridge (1970) have considered evolutionary (Friedmann) cosmologies within the framework of a series of highly conservative assumptions. These are conservative in the sense that they provide the smoothest possible projected surface distribution of radiation for a given source volume density. Even in this case, the results of Carpenter et al. (1973) imply a source density more than three times the density of galaxies as estimated by Kiang (1961). Parijskij's value would give an excess of at least two orders of magnitude.

In summary, the presently available observations indicate a remarkably isotropic radiation field on angular scales less than one degree down to a few arc minutes. Upper limits on these fine-scale intensity fluctuations are becoming small enough to be of interest in the theories of formation of large mass associations. The consequent lower limits on the space density of sources postulated to explain the $2.7 \mathrm{~K}$ background seem to completely rule out these discrete source models.

Observationally we may be on the verge of a discovery. Hopefully, within the relict radiation lurks the subtle imprint of embryonic structure in the early universe. We may be just about to detect fossil clues to our distant past; to chart a universe with barely perceivable texture, whose form should help explain the fascinating puzzle of the evolution of the highly structured cosmos we observe today. 


\section{References}

Boynton, P. E. and Partridge, R. B.: 1973, Astrophys. J. 181, 243.

Carpenter, R. L., Gulkis, S., and Sato, T.: 1973, Astrophys. J. Letters 182, L61.

Conklin, E. K. and Bracewell, R. N.: 1967, Nature 216, 777.

Hazard, C. and Salpeter, E. E.: 1969, Astrophys. J. Letters 157, L87.

Kiang, T.: 1961, Monthly Notices Roy. Astron. Soc. 122, 263.

Parijskij, Yu. N.: 1973, Astrophys. J. Letters 180, L47.

Parijskij, Yu. N. and Pyatunina, T. B.: 1970, Astron. Zh. 47, 1337.

Penzias, A. A., Schraml, J., and Wilson, R. W.: 1969, Astrophys. J. Letters 157, L49.

Smith, M. G. and Partridge, R. B.: 1970, Astrophys. J. 159, 737.

\section{DISCUSSION}

Davis: How large a correction was required in Parijskij's analysis for the confusion predicted for normal radio sources? The value quoted corresponds to $0.4 \mathrm{~m}$.f.u., which is considerably lower than the confusion level one would predict for the $140-\mathrm{foot}$ telescope at $3 \mathrm{~cm}$ wavelength.

Boynton: This information is not available in the published account of the work, and I just don't know. This is one of several questions that I had hoped to discuss with Dr Parijskij at this symposium.

Scheuer: Dr Boynton seemed to say that one can hope to detect fractional fluctuations of the order of $N^{-1 / 2}$ when there are $N$ discrete sources in the antenna beam. That is correct if we have similar sources all at the same distance, but not correct if we take the post-Copernican view that sources are distributed in depth, rather than over a hemispherical heaven. In almost all cases, the fluctuations are dominated by the brightest one or two sources in the beam, and observations of the fluctuations would not give any information about the large number of fainter sources.

Boynton: This is certainly true; the simplifying assumption of equal apparent brightness for each source is extremely conservative in the sense that the fluctuations in reality would be larger, thereby setting even more stringent limitations on the validity of the discrete source model.

\section{Small-Scale Fluctuations in the Microwave Background Radiations}

Yu. N. Parijskij (Pulkovo Observatory) and R. A. Sunyaev (Institute of Applied Mathematics, Moscow). This summary was included in the following contribution which was also read by Ya. B. Zel'dovich.

In this paper a review of observational data is presented. The measurements of Parijskij are described and the possibilities for their further development are considered. Conclusions concerning the problems of the significant contribution of discrete sources to the background radiation are given on the basis of the work of Longair and Sunyaev (Nature 223, 719, 1969). On the other hand, present measurements indicate that the observed relic radiation cannot be explained by the integrated emission of discrete sources. The most accurate estimates of small scale fluctuations were obtained at Stanford (Yu. N. Parijskij: Astron. J. USSR 50, 453, 1973), Pulkovo (Yu. N. Parijskij: Astron. J. USSR 49, 1322, 1972) and NRAO (Yu. N. Parijskij: Astrophys. J. Letters 180, L47, 1973). Recently new data were obtained at NRAO-Kitt Peak (P. E. Boynton and R. B. Partridge: Astrophys. J. 181, 243, 1973) and at JPL (Carpenter, R. L., Gulkis, S., and Sato, T.; 1973, Astrophys. J. Letters 182, L61). According to Parijskij's observations made at NRAO the dispersion of small scale fluctuations does not exceed $\Delta T \leqslant 7 \times 10^{-5} \mathrm{~K}(\lambda=2.8 \mathrm{~cm})$. The data on expected background fluctuations connected with adiabatic perturbations of density are given for comparison with this new result (see R. A. Sunyaev and Ya. B. Zel'dovich, Astrophys. Space Sci. 7, 3, 1970).

It is pointed out that secondary non-equilibrium thermalisation of pregalactic matter may lead to the smoothing out of primordial fluctuations (a large optical depth to Thomson scattering is required). On the other hand the secondary thermalisation may be inhomogeneous in space and temperature. For this reason it may cause significant fluctuations in the relic radiation.

The existence of hot intergalactic gas in clusters of galaxies should cause a decrease of the brightness temperature of relic radiation in the direction of the cluster, when $h v<k T_{r}$ (R. A. Sunyaev and Ya. B. Zel'dovich: Comments Astrophys. Space Phys. 4, 173, 1972). This effect seems to exist in the direction of Coma cluster. A positive result was obtained by Parijskij at Pulkovo in the direction of Coma cluster (see Figure 6, page 172), but additional observations are needed (see preceding references).

We should note that, in principle, the presence of gravitational waves may result in non-equilibrium relic radiation (G. Dautcourt: Astrophys. Letters 3, 15, 1969). 